\title{
Changes in circulating stem cells and endothelial progenitor cells over a 12-month period after implantation of a continuous-flow left ventricular assist device
}

Peter Ivak ${ }^{1,2,3}$, Ivan Netuka ${ }^{1,3}$, Ivana Kralova-Lesna ${ }^{1,4}$, Peter Wohlfahrt ${ }^{1}$, Jan Pitha ${ }^{1,5}$

Institute for Clinical and Experimental Medicine, Prague, Czech Republic ${ }^{2}$ Department of Physiology, Third Faculty of Medicine, Charles University, Prague, Czech Republic

${ }^{3}$ Second Department of Surgery, Department of Cardiovascular Surgery, First Faculty of Medicine, Charles University, Prague, Czech Republic

${ }^{4}$ Department of Anesthesiology and Intensive Care Medicine, First Faculty of Medicine, Charles University and Military University Hospital, Prague, Czech Republic ${ }^{5}$ Department of Internal Medicine, Second Faculty of Medicine, Charles University, Czech Republic

Submitted: 18 September 2019

Accepted: 10 June 2020

Arch Med Sci 2020; 16 (6): 1440-1443

DOI: https://doi.org/10.5114/aoms.2020.100306

Copyright @ 2020 Termedia \& Banach

\section{Abstract}

Introduction: Changes in circulating CD34+CD45low stem cells (SC) and CD34+CD45low+KDR+ endothelial progenitor cells (EPC) may reflect pathological endothelial activation. Non-pulsatile/continuous-flow left ventricular assist devices (CF-LVAD) can enhance this process. The aim of this study was to analyse the impact of 12-month CF-LVAD treatment on SC and EPC.

Methods: We analysed changes in SC and EPC from the pre-implantation period up until 12 months after implantation over 3-month intervals in 14 patients. Data from 12 patients with heart failure (HF) and from 13 healthy volunteers were used as controls.

Results: Baseline EPC were significantly higher in CF-LVAD and HF patients than in healthy controls, substantially decreasing 3 months after CF-LVAD implantation and then returning to high baseline values at 12 months.

Conclusions: Changes in circulating SC and EPC may reflect pathological endothelial activation after CF-LVAD implantation.

Key words: mechanical circulatory support, continuous flow, endothelial damage, stem cells, endothelial progenitor cells.

Long-term use of ventricular assist devices (CF-LVAD) is an effective method for end-stage heart failure management [1, 2]. However, non-pulsatile flow patterns can activate the endothelium of the systemic vasculature, thus leading to clinical complications [3]. This process can be assessed by measuring circulating CD34+CD45low stem cells (SC) and CD34+CD45low+KDR+ endothelial progenitor cells (EPC) [4]. However, long-term studies of this phenomenon are scarce. Changes in SC and EPC levels have been observed under conditions associated with endothelial damage, including cardiovascular events. SC and EPC are also involved in general reparative processes [5-7]. Significantly, reduced levels of EPC

\section{Corresponding author:} Ivan Netuka MD, PhD Institute for Clinical and Experimental Medicine

Prague, Czech Republic E-mail: ivan.netuka@ikem.cz 
have been found in advanced stages of heart failure [8].

In our previous work, we observed the biphasic response of SC and EPC 3 and 6 months after CFLVAD implantation 9. The aim of the present study was to analyse the impact of CF-LVAD on SC and EC over a substantially longer period of 12 months.

Methods. Our single-centre prospective observational study was approved by the participating institute's regulatory board and ethics committee before initiation. All patients provided their written informed consent to participate in the study.

Patients were implanted with a HeartMate II LVAD (St. Jude Medical, Pleasanton, CA), with clinical and laboratory data collected 24-48 h before and every 3 months \pm 15 days up until 1 year after implantation. Samples were analysed immediately using flow cytometry to evaluate the expression of surface antigens, as reported previously [9]. Briefly, SC and EPC were determined by flow cytometry after staining with fluorescein isothiocyanateconjugated anti-CD45, phycoerythrin-conjugated anti-CD34 and Alexa Fluor 647-conjugated antiKDR markers. Analyses were performed using the Navios EX flow cytometer, with surface antigen expression determined based on at least 1 million cells of peripheral blood. SC and EPC were also measured in 13 healthy volunteers ( 5 women, mean age: $50.1 \pm 13.5$ years) and in 12 patients with NYHA functional class III/IV heart failure (HF) ( 2 women, mean age: $52.2 \pm 7.2$ years).

A generalised linear mixed model with random intercept and gamma regression was used to analyse longitudinal changes in EPC and SC. The results of these models are presented as the estimated marginal mean with a 95\% confidence interval. Calculations were performed using SPSS 21 software (Armonk, NY, USA).

Results. Forty-five patients were originally enrolled in the study from March 2014 to March 2016. Over the 12-month period, 5 patients died and 13 were transplanted. A further 13 patients failed to complete follow-up either due to missed visit windows $(n=7)$ or technical difficulties precluding reliable measurements of SC and EPC $(n=6)$. There was no difference in baseline SC $(p=0.20)$ or EPC $(p=0.82$ ) between patients (including deceased persons), with respect to the availability of complete or incomplete long-term follow-up data. Fourteen patients completed the 12-month follow-up (including 3 women, mean age: $54.2 \pm 12.6$ years).

CF-LVAD and HF patients had significantly higher pre-implantation values of SC and EPC than healthy controls. No differences were found with respect to age, sex, aetiology of heart failure or prevalence of renal dysfunction between HF and CF-LVAD patients. As expected, the prevalence of smoking and diabetes was higher in both groups of patients than in controls (Table I).
After CF-LVAD implantation, SC had decreased at 3 months, increased at 9 months and moderately decreased at 12 months (Figure $1 \mathrm{~A}$ ). The increase between 3 months and 9 months was statistically significant $(p=0.034)$. A moderately different pattern was observed for EPC (Figure $1 \mathrm{~B}$ ), with a significant decrease at 3 months $(p=0.037)$ and an increase of borderline significance between 3 months and 9 months $(p=0.059)$. The above findings were independent of aetiology of heart failure (ischaemic vs. non-ischaemic), age and sex ( $p$ for interactions $>0.05$ ). With regard to baseline values, we found no association between SC and EPC and major clinical events (death, stroke, pump thrombosis, non-surgical bleeding).

Discussion. We observed substantial changes in circulating SC and EPC during the 12-month period following CF-LVAD implantation. SC and particularly EPC had decreased at 3 months before increasing at 6 months and 9 months. Both types of cells were moderately reduced at 12 months. We categorised changes over four periods to evaluate potential pathophysiological mechanisms. First, in relation to pre-implantation values, we found moderately higher numbers of SC and significantly higher numbers of EPC in pre-implant CF-LVAD and HF patients compared to healthy controls, potentially indicating an association between increased numbers of EPC and pathological endothelial activation caused by heart failure. Second, the reduction in both cell types 3 months after CF-LVAD implantation (values comparable to healthy controls) may not only have reflected transient endothelial deactivation (represented by EPC) but also overall improvement in tissue metabolism (represented by SC) due to dramatic improvements in haemodynamics. Third, the subsequent increase of both SC and EPC at 6 months and at 9 months may have reflected pathological endothelial reactivation caused by non-pulsatile flow, likely to have prevailed over haemodynamic stability. Fourth, the decrease of SC and EPC at 12 months may have reflected either endothelial adaptive processes or the exhaustion of endothelial regenerative capacity. It must be noted, however, that this final finding is the most speculative from a pathophysiological point of view.

The main limitations of our single-centre prospective study were the relatively low number of patients enrolled and the substantial dropout rate. Both factors impacted our ability to accurately evaluate the effects of our findings on clinical events, sex difference and other potential modifiers in this unique population. Another limitation of our study was the lack of analysis of other biomarkers associated with endothelial activation (von Willebrand factor) [10]. The main strength of our study is the employment of a long-term follow-up to assess parameters associated with vascular status in a rapidly expanding population of patients with heart 
Table I. Population descriptive statistics

\begin{tabular}{|c|c|c|}
\hline Parameter & $\begin{array}{l}\text { Healthy controls } \\
(n=13)\end{array}$ & $\begin{array}{l}\text { Type/effect } \\
(n=12)\end{array}$ \\
\hline Age [years] & $53.0[41.0-58.0]$ & $52.0[47.0-57.5]$ \\
\hline Sex (male), $n(\%)$ & $8(61.5 \%)$ & $12(100 \%)$ \\
\hline Smoking, $n(\%)$ & $1(7.7 \%)$ & $1(8.3 \%)$ \\
\hline Diabetes mellitus, $n(\%)$ & $0(0 \%)$ & $6(50 \%)^{*}$ \\
\hline Ischemic cardiomyopathy, $n$ (\%) & NA & $5(41.7 \%)$ \\
\hline Creatinine $[\mu \mathrm{mol} / \mathrm{l}]$ & NA & $99[86-125]$ \\
\hline Urea [mmol/l] & NA & $8.7[6.8-11.2]$ \\
\hline Haemoglobin $[\mathrm{g} / \mathrm{l}]$ & NA & 148.5 [136.0-161.0] \\
\hline SC baseline [cells/ml]: & 1889 (1005-3551) & $3,061(2,315-4,047)$ \\
\hline 3 months & NA & NA \\
\hline 6 months & NA & NA \\
\hline 9 months & NA & NA \\
\hline 12 months & NA & NA \\
\hline EPC baseline $[$ cells $/ \mathrm{ml}]$ : & $91(60-136)$ & $175(115-268)^{*}$ \\
\hline 3 months & NA & NA \\
\hline 6 months & NA & NA \\
\hline 9 months & NA & NA \\
\hline 12 months & NA & NA \\
\hline
\end{tabular}

A

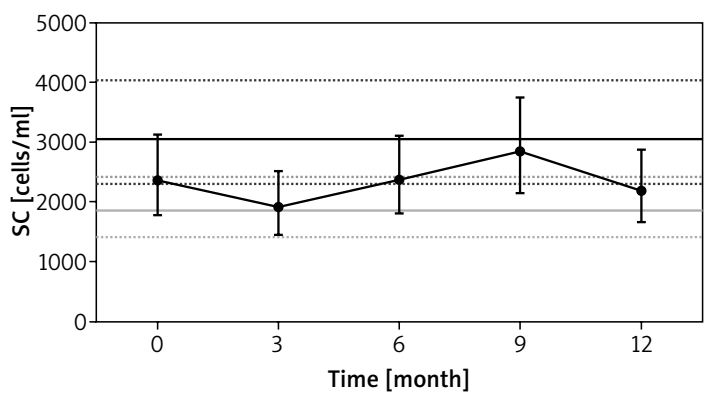

B

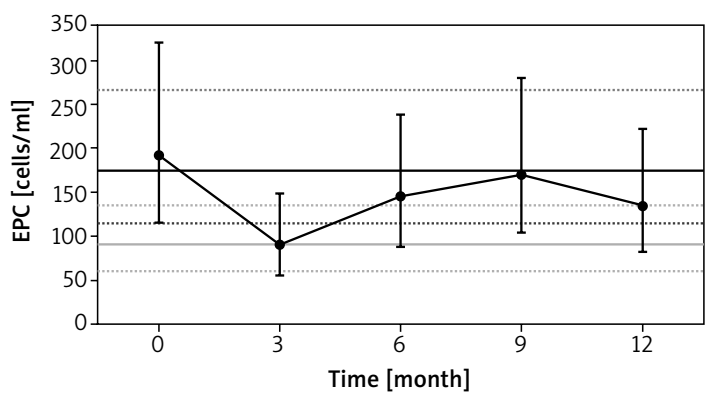

Figure 1. Longitudinal changes of circulating CD34+CD45low stem cells (A) and CD34+CD45low+KDR+ endothelial progenitor cells (B)

Solid grey line - estimated marginal mean for healthy controls; solid black line - estimated marginal mean for advanced heart failure patients; dashed lines represent 95\% confidence interval. SC - CD34+CD45low stem cells, $E P C-C D 34+C D 4510 w+K D R+$ endothelial progenitor cells.

failure treated with CF-LVAD. An association between these parameters and clinical events needs to be established by larger prospective studies.

In conclusion, circulating markers of vascular damage, particularly circulating CD34+CD45low stem cells and CD34+CD45low+KDR+ endothelial progenitor cells, can be a useful tool in assessing the effects of mechanical heart support on vasculature as well as on potential serious clinical events.

\section{Acknowledgments}

This study was supported by the Ministry of Health of the Czech Republic under grant no. 16-27630A. All rights reserved. 


\section{Conflict of interest}

$\mathrm{PI}$ is a consultant for Abbott and CARMAT S.A. In addition to receiving grant funds, IN is a consultant and serves on advisory boards for Abbott, Carmat S.A., EvaHeart and LeviticusCardio Ltd. PI, IN, IKL, PW and JP all received institutional grant support from Abbott.

\section{References}

1. Horn P, Kelm M, Westenfeld R. Integration of medical therapy and mechanical circulatory support in the management of acute heart failure. Arch Med Sci 2016; 12: 1317-23.

2. Kirklin JK, Xie R, Cowger J, et al. Second annual report from the ISHLT Mechanically Assisted Circulatory Support Registry. J Heart Lung Transplant 2018; 37: 685-91.

3. Witman MA, Garten RS, Giffort JR, et al. Further peripheral vascular dysfunction in heart failure patients with a continuous-flow left ventricular assist device: the role of pulsatility. JACC Heart Fail 2015; 3: 703-11.

4. Manginas A, Tsiavou A, Sfyrakis P, et al. Increased number of circulating progenitor cells after implantation of ventricular assist devices. J Heart Lung Transplant 2009; 28: 710-7.

5. King TF, McDermott JH. Endothelial progenitor cells and cardiovascular disease. J Stem Cells 2016; 9: 93-106.

6. Pokrywka A, Zembron-Lacny A, Baldy-Chudzik K, et al. The influence of hypoxic physical activity on cfDNA as a new biomarker of vascular inflammation. Arch Med Sci 2015; 11: 1156-63.

7. Kavala AA, Turkyilmaz S. Autogenously derived regenerative cell therapy for venous leg ulcers. Arch Med Sci Atheroscler Dis 2018; 3: 156-63.

8. Koller L, Hohensinner P, Sulzgruber P, et al. Prognostic relevance of circulating endothelial progenitor cells in patients with chronic heart failure. Thromb Haemost 2016; 116: 309-16.

9. Ivak P, Pitha J, Wohlfahrt P, et al. Biphasic response in number of stem cells and endothelial progenitor cells after left ventricular assist device implantation: a 6-month follow-up. Int J Cardiol 2016; 218: 98-103.

10. Netuka I, Kvasnička T, Kvasnička J, et al. Evaluation of von Willebrand factor with a fully magnetically levitated centrifugal continuous-flow left ventricular assist device in advanced heart failure. J Heart Lung Transplant 2016; 35: 860-7. 